University at Albany, State University of New York

Scholars Archive

2012

\title{
Finding Citations to Social Work Literature: The Relative Benefits of Using Web of Science, Scopus, or Google Scholar
}

Elaine M. Lasda Bergman

University at Albany, State University of New York, elasda@albany.edu

The University at Albany community has made this article openly available.

Please share how this access benefits you.

Follow this and additional works at: https://scholarsarchive.library.albany.edu/ulib_fac_scholar

Part of the Scholarly Communication Commons, and the Social Work Commons

\section{Recommended Citation}

Lasda Bergman, Elaine M., "Finding Citations to Social Work Literature: The Relative Benefits of Using Web of Science, Scopus, or Google Scholar" (2012). University Libraries Faculty Scholarship. 18.

https://scholarsarchive.library.albany.edu/ulib_fac_scholar/18

This Article is brought to you for free and open access by the University Libraries at Scholars Archive. It has been accepted for inclusion in University Libraries Faculty Scholarship by an authorized administrator of Scholars Archive.

Please see Terms of Use. For more information, please contact scholarsarchive@albany.edu. 


\section{Finding Citations to Social Work Literature: The Relative Benefits of Using Web of Science, Scopus, or Google Scholar}

\section{$\underline{\text { Introduction }}$}

Google Scholar and Scopus are relatively new tools available to scholars for identifying cited and citing references to a given research article. The present study compares these tools to the longstanding, established citation indexes available through the Web of Science database, focusing on social work and social welfare research. To do this, the citations to articles in five leading social welfare journals were obtained from Web of Science, Scopus, and Google Scholar. The patterns of citing references contained in these databases were compared and contrasted to determine the utility and robustness of social welfare coverage in each source of citation data.

The citation indexes found in Web of Science (Science Citation Index, Social Science Citation Index and Arts and Humanities Index) have existed since the 1970s, when they were print resources. On the other hand, Google Scholar and Scopus both appeared in the first decade of the $21^{\text {st }}$ century, and contain overlapping content with Web of Science and each other, but as is demonstrated in the literature review, there are often unique items in each source.

Because of its interdisciplinary nature, research on social work and social welfare topics may appear in sources that are based in various academic disciplines. As a result, it is not immediately clear what, if any, advantages each citation indexing source may have in covering social welfare research. This study seeks to determine: if there are any patterns in the overlap of citing reference coverage for scholarly works in Scopus, Google Scholar and Web of Science; to what extent each resource provides unique citing references for these works; and if there are any significant patterns to the unique references in each index. 
Thyer noted that the interdisciplinary nature of social work as a profession and a disciple creates obstacles to creating unique knowledge, concluding that the knowledge generated in social work research should be the development of "problem-specific" knowledge. ${ }^{1}$ By this, he means that "a focus on the development, testing, and validation of psychosocial interventions [should] be the primary scholarly mission" of the field of social work, and that these interventions can be applied in a variety of human services settings. ${ }^{2}$ Thus, the research domain of social work rests in the application of interventions that are based on what Thyer terms as "subject matter" research from other disciplines.

Strothmann and others before her have also noted that social work research is both multidisciplinary and interdisciplinary, and have sought to determine characteristics and aspects of essential social work literature. Strothmann, who is the most recent of these scholars, found that researchers use a wide variety of social science, medical and "hard" science resources. ${ }^{3}$ Pierce has noted that bibliometric analysis poses certain challenges when dealing with interdisciplinary fields: the literature, perspectives, and theories are less cohesive and there are fewer distinct boundaries to the field. ${ }^{4}$ The varying results of previous analyses of citation databases do not allow the researcher to reliably predict the relative strengths of each tool for use in analyzing social welfare research patterns.

This study is of significance to scholars in Information Studies in that it will shed additional light on the effectiveness of the citation databases in mapping scholarly communication; it will also be of significance to scholars in Social Work and Social Welfare interested in the relative strengths of these databases as they pertain to their field of research.

\section{Review of the Literature}

Bibliometrics in Social Work Research 
Bibliometrics in social work has been a somewhat recent phenomenon relative to its use in other disciplines. As recently as 2006, a special edition of the journal Social Work in Health Care was published as a monograph with a number of essays introducing the concepts of bibliometrics to social work researchers and discussing the applications of bibliometrics to research in the field. ${ }^{5}$ The monograph includes an introductory literature review of citation analyses performed on social work topics; a discussion of how tracing citation patterns can indicate the impact of a scholar's idea; essays related to the impact of bibliometric indicators on promotion, tenure, and evaluation of faculty; clarifications that need to be made to bibliometrics to assure a credible measurement of "merit"; an essay identifying factors such as publication lag time and the quality of the peer review process that affect bibliometric indicators; and concludes with an essay about the contribution bibliometrics makes to tracing scholarly communication patterns and the evolution of knowledge about a topic. The application of bibliometric methodologies to social work research has resulted in some interesting findings. Strothmann found that although there is a core body of essential journals falling squarely within the discipline of social work, research also incorporates resources from areas falling outside of the social sciences, such as psychiatry and medicine. ${ }^{6}$ Others have performed citation analysis on social work faculty publications, finding that social work scholars will be cited more frequently if they publish in a non-social work journal. ${ }^{7}$

At the onset of bibliometric research in social work there were concerns regarding the use of the ISI Indexes (now Web of Science) due to the fact that they emphasized the "hard" sciences; and also because citation patterns in the social sciences are not comparable to those in the hard sciences and therefore should not necessarily be measured in the same manner. ${ }^{8}$ Furr did an analysis of the utility of the Journal Impact Factor (JIF) with regard to social work research. He found that the Social Science Citation Index did not include nine journals deemed to be essential to the field. As a result, according to Furr, the JIF is not an accurate measure of the impact of social work journals. ${ }^{9}$ 
Comparisons of Web of Science, Scopus, and Google Scholar

Comparison of citation databases has been the subject of a variety of studies using various bodies of research, and applied to various academic disciplines and subspecialties.

In her 2010 book about citation analysis which provided general commentary on the coverage of Google Scholar, Web of Science, and Scopus titled Publish or Perish, Harzing asserted that for the social sciences and humanities Google Scholar has an "advantage" in citation count over Web of Science and to a lesser degree Scopus. Furthermore, she finds that the journal coverage of Google Scholar is superior to both Web of Science and Scopus. ${ }^{10}$ However, Harzing based this conclusion on having studied a single scholar in each of the following disciplines: Business, Cinema Studies, Education, Linguistics, and Political Science. It is unclear, therefore, whether this sample is truly representative of a greater pattern in all of the social sciences and humanities.

Aside from the broad generalizations in Harzing's book, there have been numerous studies comparing coverage of the three citation databases with regard to academic subspecialties, journal coverage, scholarly output and other measures which vary from the general patterns espoused by Harzing.

Meho, with several different coauthors, has done several studies in this regard, comparing citation counts, rankings and coverage for various groups of library and information science scholars ${ }^{11}$. In these studies he found that citation-based rankings of scholars, (and presumably journals) are affected by including Scopus with Web of Science findings, but less so when adding Google Scholar. For accurate citation counts, all three resources must be used. Meho and Sugimoto found that in ranking scholarly productivity, when small scale citation patterns are sought, such as for journals, conference proceedings, and institutions, both Web of Science and Scopus should be utilized to get an accurate ranking, because the rankings differ greatly between these two sources. On the other hand, when looking at larger scale categories such as ranking research domains or countries, Scopus may be used 
interchangeably with Web of Science. ${ }^{12}$ When studying human-computer interaction scholars, Meho and Rogers found Scopus to be an acceptable substitute for Web of Science. ${ }^{13}$ Another scholar analyzed the coverage of Web of Science and Google Scholar for a corpus of earth science faculty and found that 85\% of the citations in Web of Science were located in Google Scholar, the two provide similar h-index results (a measurement of scholarly output), and other rankings demonstrate similar results. ${ }^{14}$ With regard to citation count, the author determined that the large number of citations in Google Scholar not included in Web of Science constitute a "long tail" of results of minor importance. ${ }^{15}$

Tracking the coverage of journals or the citations to journal articles is another frequent means of comparing Web of Science, Scopus and Google Scholar. Levine-Clark, et al., compared the three citation databases for citing references to top articles from fifteen business and economics journals. ${ }^{16}$ They found that Scopus produced slightly higher citation counts than Web of Science, and note that the citations to non-scholarly resources found in Google Scholar can demonstrate influence or impact. They found that Google Scholar is a reasonable alternative for business and economics citation searching if the proprietary fee based resources are not available. In a comparison of articles in major medical journals in Google Scholar and Web of Science, Kulkarni, et al. found variations in coverage for certain subgroups of articles (e.g., industry funding, devices, group authorship) but all had higher citations in Web of Science and Scopus than in Google Scholar. ${ }^{17}$ Haddow looked at Web of Science for Australian social science journals and found that Scopus had more citations than Web of Science and covered more of the journals. This led him to conclude that an Australian government research assessment protocol was not reflective of citation patterns of this same body of research found in the citation databases. ${ }^{18}$ Other analyses compared the citation databases to subject specific databases. Norris and Oppenheim compared coverage of references citing Great Britain's Research Assessment Exercises and the International Bibliography of the Social Sciences in Scopus, CSA Illumina, and Web of Science. ${ }^{19}$ They 
found that Scopus had the best coverage of their interdisciplinary sample, and that both Scopus and Web of Science had better coverage than CSA Illumina. Bornmann, et al. compared the coverage of a body of articles in the three citation databases to Chemical Abstracts and found that all provided very similar results. ${ }^{20}$

Still others have compared the coverage of other miscellaneous resources such as comparing the number of citing references found for a single book ${ }^{21}$ and the scholarly output of countries and institutions. ${ }^{22}$ Google Scholar was found to be superior in generating the highest citation count for the book. The ranking results of countries and institutions in Scopus and Web of Science were found to be correlated. Still another researcher created a literature review comparing Google Scholar and Web of Science as a means of facilitating the instruction of both sources in information literacy instruction sessions. $^{23}$

The variations in breadth and depth of these studies do not indicate clear patterns that can be immediately assumed to represent the coverage of citing references to social work sources in each of the three citation databases. Furthermore, as has been shown, social work research relies on an interdisciplinary blend of research; therefore the results of previous studies on coverage of various social science disciplines may not be directly analogous to the results to be garnered from studying social work literature in its own right.

\section{Methodology}

Before beginning any citation analysis, one must determine the body of research that will be used as a basis for collecting citing references. A common method is to choose top journals based on a citationbased ranking metric, most typically the Journal Impact Factor (JIF). The intent of this study was to choose journals from a ranking that was not, in and of itself, predicated on a bibliometric indicator, because those scores are derived from the data supplied by the citation databases under analysis in this 
study. The citation information used to compute the Journal Impact Factor (JIF) is contained in the Science and Social Science Citation Indexes of Web of Science. SCImago Journal Rank (SJR) and Source Normalized Impact per Paper (SNIP) use the data from the Scopus product, and there are other metrics computed by the downloadable tool Publish or Perish that provide similar rankings based on the data found in Google Scholar. Sellers, et al. undertook a comprehensive study of social work journal quality, and among other metrics, provides a table ranking key social work journals according to their reputation among 556 faculty members of accredited schools of social work ${ }^{24}$. The article by Sellers, et al. provided an alternate ranking system of top journals in the field, based on scholar perception. Unfortunately, this ranking was not completely uninfluenced by a citation metric, as the JIF was used as one of the starting points to compile the journal list for the social work faculty to evaluate. The researchers also consulted a list of journals published in a previous survey, which were apparently derived from reviewing a variety of resources with information about scholarly journals in order to identify titles which met certain criteria for inclusion. ${ }^{25}$ In addition, Sellers, et al. offered the faculty an option to add additional journals not included in the original survey, but there was no clear pattern to those suggested by respondents, and they were not used for the final analysis.

The present study used the top 5 journals of Sellers, et al.'s study that had the highest mean overall quality scores. ${ }^{26}$ In other words, these were the top five journals ranked highest in overall quality by the 556 faculty members surveyed. These journals were: Social Service Review, Social Work Research, Journal of Social Service Research, Child Abuse and Neglect, and Research on Social Work Practice. The analysis focused on articles and other materials published in the selected journals in the year 2005. Scopus, Web of Science, and Google Scholar were all searched to find references citing the materials published in 2005 in these journals. The year 2005 was chosen because it allowed sufficient time for the publications to be discovered, disseminated, and be cited by other researchers. At the same time, the 
citing references to these journals will be relatively recent (having been published after 2005), therefore a realistic reflection on what publications are being used by current social work researchers.

Both the Science Citation Index and the Social Science Citation Index were searched in Web of Science; likewise, the searches in Scopus were not limited by subject areas. The citing reference data from Scopus and Web of Science was exported into Microsoft Excel, and then transferred to Microsoft Access, which was used to analyze results. The citing references in Google Scholar were retrieved manually and entered into an Excel spreadsheet. This proved beneficial in terms of identifying the source type of each citing reference, as there does not appear to be a way to automate the capture of metadata on document type or format of the citing reference in Google Scholar; furthermore, the many citing references which were a non-traditional source type (e.g., a source type other than journal article, book, or conference proceeding) were easily identified.

Citing references from any material published in the sample journals during the year 2005 were included in the data capture. To keep the capture process manageable, no effort was made to eliminate editorials, book reviews or other non-peer reviewed material. In addition to manageability, the investigator felt that this approach would accurately capture the patterns of citing the sample journals in their entirety. This gives the analysis a fuller picture of what Scopus, Web of Science, and Google Scholar contain in terms of citing reference information.

The citing reference data for each journal was imported into a Microsoft Access database to facilitate analysis. Queries were generated to compare the coverage of citing references in all three databases for the journals together, and individually to determine any patterns. Once this data was collected and analyzed, it was evident that a closer look at the Google Scholar results would provide additional insight into the nature of coverage of that database.

Problems with Data Collection form Google Scholar 
In collecting data from Google Scholar, results that were bad links or for which there was no reference information were discarded. Some issues arose when assigning the source types of the citing references. There were a significant number of citing references which were not journal articles, but instead web pages, word processing documents, and PDF files. Sometimes the PDF files and Word documents retrieved contained no direct statement about publisher information, and these were generally deemed to be unpublished manuscripts. Sometimes resources retrieved from Google Scholar were draft versions of research that was published in a scholarly journal at a later date and the draft and officially published version were sometimes listed as two separate results. In these cases, only the officially published versions of the article were counted as citing references. Furthermore, items designated as research papers, working papers, or reports, were all classified as "reports" based on the premise that all contained research findings and although some were published, none appeared in a scholarly journal.

Another problematic issue in Google Scholar was the handling of foreign language documents. In some cases, if a citing reference was to a document that was in a foreign language it was easy to discern if it was a journal article, doctoral dissertation, etc. whereas other times the source type/format could not be determined. All of these items were categorized as source type "foreign language" rather than make a distinction between the foreign language materials of known format and those of unknown format. Quantifying the foreign language resources as a whole was important, and there was presumed to be a similar ratio of formats in the foreign languages to those in found in English and therefore the results of the analysis would still be proportionally accurate.

\section{$\underline{\text { Results and Analysis }}$}

Database Coverage of Citing References 
[Figure 1]Taking in the aggregate the data from all five journals, a total of 1,741 citing references were collected from Web of Science, 2,126 citing references were collected from Scopus, and 3,272 were collected from Google Scholar, for a total of 7139 results before removing duplicates. When results for all three databases were combined and overlapping citations were taken into account, there were 4,308 unique citing references in total.

A total of 961 citing references were found in all three sources, or $22.3 \%$ of all unique citations. This constituted roughly half (55.2\%) of the total citing references in Web of Science as well as Scopus (45.2\%). By contrast, just under one third (29.4\%) of the citing references in Google Scholar were found in all three sources.

Citing references that were found in both Scopus and Web of Science, but not Google Scholar constituted 502 items or $11.7 \%$ of all unique citations. These 502 citations constituted $28.8 \%$ of the items in Web of Science and $23.6 \%$ of the items in Scopus.

Citing references that were found in both Scopus and Google Scholar but not Web of Science constituted 324 items or $7.5 \%$ of all unique citations. These represented $15.2 \%$ of the items in Scopus and $9.9 \%$ of the items in Google Scholar.

Citing references that were found in both Web of Science and Google Scholar but not Scopus constituted 81 items or $1.9 \%$ of all unique citations. This represented $4.7 \%$ of the items in Web of Science and $2.5 \%$ of the items in Google Scholar.

Google Scholar had far and away the greatest number of citing references not included anywhere else, a total of 1904 or $44.2 \%$ of all unique citations. Scopus was the sole source for 339 of the 4308 unique citations, or 7.9\%, and Web of Science was the only source for 197 or $4.6 \%$.

Variations in Format/Document Type 
[Figure 2]The formats of the citing references (source types) were compared among databases. In Scopus, of the 2126 total citing references retrieved, 1,782 or $83.8 \%$ were journal articles, 248 or $11.7 \%$ were reviews, and 96 or $4.5 \%$ were miscellaneous items such as conference papers, editorials, notes, letters, and short surveys.

In Web of Science, 1735 (99.7\%) of 1,741 total citing references were journal articles and the remaining $6(0.4 \%)$ were labeled series. Although the Web of Science documentation explains that "series" is "book in a series," it was felt that this explanation was not clear. ${ }^{27}$ Upon examining these citations, it appeared that they consisted of scholarly article-length research that appeared in annual compendia as opposed to journals. Although Web of Science lists only three other possible publication types (conference, book, or patent), none of these publication types appeared in these results.

Google Scholar had a much more diverse array of citing references. Only 1,951 or $59.6 \%$ of the 3272 citing references retrieved were scholarly journal articles; 443 or $13.5 \%$ were dissertations, masters' theses, or bachelor's theses; 318 or $9.7 \%$ were books, and 281 or $8.6 \%$ were foreign language materials. The remaining 279 or $8.5 \%$ constituted a wide variety of miscellaneous sources such as reports, course syllabi, unpublished manuscripts, reviews, presentation slides, blogs, and websites.

It is interesting to note that Google Scholar contained a total count of 1,951 citing references that were scholarly journal articles. Although this was only roughly $60 \%$ of the total citations in Google Scholar, it is only slightly higher than the total count of scholarly journal articles in Scopus (1782) and Web of Science (1735).

The five journals selected for review in this study were cited in widely varying degrees. Some of this variation could be attributed to the differing frequencies of publication of each journal: Child Abuse and Neglect is published twelve times per year, Research on Social Work Practice is published bimonthly, 
Journal of Social Service Research is published five times per year, and Social Work Research and Social Service Review are only published quarterly.

The Journal of Social Services Research, ranked third on the Sellers, et al. list, had substantially lower citation rates than all other journals considered, despite being published five times per year. Upon further inspection, it was found that prior to 2010 this journal was published quarterly and sometimes irregularly. Volume 31 contains issues published in the years 2004-2006, with only two issues published in 2005. Therefore, the extremely low citation rate from all sources for this journal is presumed the result of the very small number of issues available for the selected publication year of study.

On the other hand, Child Abuse and Neglect ranked fourth by the scholars polled in the study by Sellers, et al. actually had the highest combined number of citing references when duplicates were eliminated (2715). Research on Social Work Practice was second highest with 749 citing references. Social Service Review ranked third (408), but was comparable to Social Work Research (361). Journal of Social Service Research had 75 total citing references, this may be due at least partially to the fact that this journal is only published quarterly and there are fewer articles to cite. [Figure 3] This is substantially different than the ranking in Sellers, et al.'s study in which Social Service Review was ranked first, followed by Social Work Research, Journal of Social Service Research, Child Abuse and Neglect, and Research on Social Work Practice, in that order. [Figure 4]

\section{Comparison of Database Coverage of Citing References per Journal}

The percentage of citing references to each journal was considered separately to determine whether the patterns of citation coverage for each database were consistent (e.g., is the percentage of citations retrieved from each citation database consistent from journal to journal), or if the percentage of citing references retrieved for each journal varied. For breakdowns by source type, see [Figure 5]. Coverage of each journal in the three databases varied somewhat. [Figure 6] Journal of Social Service Research had 9 
citing references appearing in all three databases out of the 75 unique citations to this journal, or $12.0 \%$. For Social Work Research, 85 or $23.5 \%$ of the 361 unique citations were found in all three databases; Social Service Review had 86 or $21.1 \%$ of the 408 unique citations to this journal. Research on Social Work Practice had 141 or $18.3 \%$ of the 749 unique citations. For Child Abuse and Neglect, 640 or $23.6 \%$ of the 2715 unique citations were found in all three databases.

Of the citing references found in Scopus and Web of Science, Journal of Social Service Research had 4 citing references or $5.5 \%$ of all unique citations. Social Work Research had 46 citing references or $12.7 \%$. Social Service Review had 48 citing references or $11.8 \%$. Research on Social Work Practice had 89 citing references or $11.9 \%$. Child Abuse and Neglect had 315 citing references or $11.6 \%$.

Of the citing references found in Scopus and Google Scholar, Journal of Social Service Research had 12 citing references or $16.0 \%$ of all unique citations, Social Work Research had 41 citing references or 11.4\%. Social Service Review had 25 citing references or 6.1\%. Research on Social Work Practice had 83 citing references or $11.1 \%$. Child Abuse and Neglect had 163 citing references or $6.0 \%$.

Of the citing references appearing in Web of Science and Google Scholar, Journal of Social Service Research had 2 citing references or $2.7 \%$ of all unique citations, Social Work Research had 10 citing references or $2.8 \%$. Social Service Review had 10 citing references or $2.5 \%$. Research on Social Work Practice had 11 citing references or $1.5 \%$. Child Abuse and Neglect had 48 citing references or $1.8 \%$.

The percentage of citing references that were unique to each database was also examined on a per journal basis. For Google Scholar, Journal of Social Service Research had 41 citing references or $54.7 \%$ of all unique citing references retrieved for this journal. Social Work Research had 140 citing references or 38.8\%. Social Service Review had 212 citing references or 52.0\%. Research on Social Work Practice had 324 citing references or $43.3 \%$. Child Abuse and Neglect had 1187 citing references or $43.7 \%$. 
For Web of Science, Journal of Social Service Research had 7 citing references or $9.3 \%$ of all unique citations, Social Work Research had 18 citing references or 5.0\%. Social Service Review had 9 citing references or $2.2 \%$. Research on Social Work Practice had 27 citing references or $3.6 \%$. And Child Abuse and Neglect had 136 citing references or $5.0 \%$.

For Scopus, Journal of Social Service Research had no citing references that did not appear any other database. Social Work Research had 21 citing references or 5.8\%. Social Service Review had 18 citing references or 4.4\%. Research on Social Work Practice had 74 citing references or $9.9 \%$. Child Abuse and Neglect had 226 citing references or $8.3 \%$.

When each journal is considered separately, the percentages of citing references retrieved show a pattern consistent to that which emerged when the citing references were considered in the aggregate for each database. In other words, the relative percentage of citing references retrieved for one journal that appear in one, two or all three databases is roughly consistent to the percentage of citing references retrieved for each of the other journals. For example, one can expect to retrieve around half of the citing references to each journal if one uses only Web of Science and Scopus. The only instance where the percentage of coverage appears inconsistent is the citing references to Journal of Social Service Research, the journal which only had two issues published in the year 2005. In this case, Google Scholar provided over half the citing references retrieved (54.7\%). It is reasonable to presume that this high percentage of citing references is composed of non-traditional document types. Web of Science and Scopus, which retrieved correspondingly lower percentages retrieved, primarily collect citing references from journal articles.

\section{Considerations Regarding Google Scholar Coverage}

Earlier, it was noted that 1,951 or $59.6 \%$ of all citations found in Google Scholar were journal articles, and that number is only slightly higher than the total number of citations to journal articles in Scopus or 
Web of Science. For Social Work Research, 39 scholarly journal articles were retrieved from only from Google Scholar. This represents $10.8 \%$ of the citing references unique to this journal, or $1.2 \%$ of the total citations retrieved from Google Scholar. Google Scholar was the sole source for 80 scholarly articles which cited Social Service Review, constituting $19.6 \%$ of the total unique citations to this journal, and 2.4\% of all citations in Google Scholar. Journal of Social Service Research had 22 citing references unique to Google Scholar that were scholarly journal articles. These constituted $29.33 \%$ of the unique citations to this journal, and $0.7 \%$ of the total citations retrieved from Google Scholar. Research on Social Work Practice had 111 citing references from scholarly journal articles appearing only in Google Scholar. This constituted $14.8 \%$ of the total unique citations to this journal, or $3.4 \%$ of the citations retrieved from Google Scholar. Child Abuse and Neglect had 356 citing references from scholarly journal article found only in Google Scholar (out of 1187 unique Google Scholar citing references), constituting $13.1 \%$ of all unique citations to this journal, and $10.9 \%$ of all citations retrieved from Google Scholar. Therefore Google Scholar provided roughly $10 \%-20 \%$ of citing references in scholarly journal format that were not found in the proprietary databases.

\section{Patterns in Citation Count}

The interval between the citation count of the highest cited journal (Child Abuse and Neglect) and the other four journals was computed and the results for each database were plotted on a graph [Figure 7]. The difference in citation counts between Child Abuse and Neglect and each other journal shows a roughly proportional set of intervals between citation counts in Scopus and Web of Science. The interval rankings, from lowest to highest for both of these databases, is Research on Social Work Practice, Social Work Research, Social Service Review, Journal of Social Service Research. Google Scholar however, has a lower interval for Social Service Review than for Social Work Research. Google Scholar consistently has the highest intervals, Scopus second highest, and Web of Science third highest. When only the scholarly 
journal citations from Google Scholar are considered, the intervals between citation counts are much closer to Scopus and Web of Science, but follow a more erratic pattern. There is a greater difference between the intervals computed for each journal, indicating a coverage pattern that is not consistent with that of the proprietary databases. As with the interval for all Google Scholar citations, the interval for Social Service Review is lower than that for Social Work Research, however, the difference is more pronounced when only the scholarly journals are counted. This may be reflective of how journals and journal articles are chosen for inclusion in each citation database. Scopus claims to include 18,500 peerreviewed journals as well as hundreds of trade publications, book series, and conference proceedings. ${ }^{28}$ Inclusion in Scopus is determined by the Scopus Content Selection and Advisory Board, consisting of fourteen experts in a variety of subject disciplines. ${ }^{29}$ Web of Science states that it covers over 12,000 "top-tier" journals and inclusion in the database is determined by editors at Thomson Reuters. ${ }^{30}$ Google Scholar, on the other hand, does not have any systematic means of evaluating journals for inclusion - by its very nature it cannot-- because it is first and foremost a web search engine, it simply captures what has been published on the World Wide Web. Thus, the non-systematic means of collecting citation data provides no consistent pattern or system by which citing references are included or retrieved from Google Scholar; whereas the inclusion and retrieval of citing references in Scopus and Web of Science is systematic in nature, and therefore a similar pattern of citation coverage is demonstrated.

Citing References in Foreign Languages

Foreign language materials constituted 281 or $6.5 \%$ of the 4308 total unique citations from all databases and $8.6 \%$ of the total number of citations retrieved from Google Scholar (3272). The foreign languages represented were very diverse; materials in all of the following languages cited at least one of these social welfare journals: French, Dutch, German, Chinese, Korean, Japanese, Polish, Portuguese, Turkish, Russian, and Finnish. With the trend towards greater globalism and international research, it 
has become more important to be able to trace the international impact or influence of a journal, institution, author, or individual article.

\section{Discussion}

Limitations of This Study

While the results and analysis of this data provide some interesting insights into the coverage of the citation databases for social welfare resources, there are some limitations which must be taken into consideration. Primarily, the Sellers, et al. article from which the key social work journals were identified was published in 2004. If such a study were undertaken in the current year, the ranking may be substantially different and may include journals that were not in publication eight years ago.

Updating the findings of this research based on the opinions of current social welfare scholars may well be a topic ripe for further study; in fact, the Sellers, et al. article was an update of an earlier article from 1994. ${ }^{31}$ As previously stated, most ranked listings of journals are based upon citation metrics (Journal Impact Factor being the most prevalent). The 2004 Sellers article is the most current ranking which relies on a metric other than citation activity - namely, perceived value determined by a survey of social work scholars. However it is likely that these findings would be similar if a more current list were used, due to the fact that journal ranking is not a factor in the citation coverage of Web of Science, Scopus, or Google Scholar.

Another limitation to this study is the number of journals analyzed. Harvesting the citing references from Google Scholar for these five journals took approximately 140 hours in total. Adding additional journal titles to the analysis would have been preferred but was not feasible given the resources available (student assistant, grant funding) for this project. 
Citations in foreign languages posed other issues when comparing Google Scholar to the other databases. There appeared to be few, if any citing references in Web of Science or Scopus from foreign language sources retrieved for this search. However, the metadata captured from these databases did not include a language field. At the same time, it is possible that there were bibliographic records in both English and the native language listed as separate results in Google Scholar. Due to the diverse array of foreign languages retrieved and a lack of knowledge of these languages means that a slight possibility exists that some articles may have been double counted in cases where bibliographic data of the citing reference for a single source was presented in the native language in one instance, and translated into English in another instance.

Furthermore, others have shown that subspecialties of various disciplines can have different rates of coverage in each database. ${ }^{32}$ Four of the five journals considered here are certainly very centered in social welfare scope (Research on Social Work Practice, Social Work Research, Social Service Review, Journal of Social Service Research), but Child Abuse and Neglect has a different topic focus, and at the same time has vastly broader usage. As all subspecialties in social welfare cannot be considered by a study of this scale, ascertaining the coverage of citations for research in certain interdisciplinary topics which rely more heavily on, for example, medical or education research may result in different results. Another consideration is that this study only measures the raw citation count. Other metrics, such as those based on eigenvector analysis (where some citing references in a network are weighted more heavily in the computation due to greater use) may result in different rankings and provide a more robust picture of citation patterns in this subject. ${ }^{33}$ Furthermore, other measurements may be useful to consider, such as the h-index and variations that account for different citation patterns and rates over time. $^{34}$

Ranking of Journals Based on Citation Count 
When ranking the journals by highest number of citations to lowest, the rank is identical in Scopus and Web of Science to that of the ranking produced when data for all three databases are taken in the aggregate: Child Abuse and Neglect received the highest citation count in both Scopus and Web of Science, followed by Research on Social Work Practice, Social Work Research, Social Service Review, and finally, Journal of Social Service Research, in that order. Google Scholar had similar results; however Social Service Review ranked above Social Work Research. However, this may not be significant, because in all comparisons, these two journals had relatively close citation counts.

Child Abuse and Neglect was cited in the widest variety of document types. [Figure 5] This is understandable due to the substantially larger number of citing references in total for this journal. Also, this journal has an audience that is international in scope, as it is the official journal of the International Society of Prevention of Child Abuse and Neglect and is of interest to psychologists and other scholars and practitioners in various social sciences. The other four are published less frequently and focus more squarely on aspects of social work and social services.

\section{Best Use of Citation Databases}

Tracing scholarly communication is a meritorious pursuit. Scholars cite other research for many reasons; one author contends that citing a source is in some respect, a social process rather than a scientific one. ${ }^{35}$ The motivation to cite a source may be: "conceptual," "contrastive," "methodological," "negational," "perfunctory," or "persuasive." ${ }^{36}$

The results of this study show that for the social welfare journals studied, Scopus generally provides higher citation counts than Web of Science, but that a ranking from highest to lowest citation count in each database results in similar outcomes. On the other hand, total Google Scholar citations do not follow an identical ranking, although the ranking is very close. When only Google Scholar journal article citations are considered, the ranking diverges further. Therefore, Web of Science and Scopus may likely 
provide correlated results in terms of ranking based on citation counts for other journals. The pattern established when considering citing references to all five journals in the aggregate are replicated when the citing references to each journal are considered separately. The results of this study do not affirm Harzing's findings that Google Scholar has a consistent advantage in coverage of the social sciences. ${ }^{37}$

Taken together, Web of Science and Scopus will only retrieve around half of the unique citing references retrieved from searching all three databases. Therefore if one is looking for a high citation count or a complete list of citing references for research purposes, it is wise to consult all three sources and remove duplicates, which is an unfortunately time consuming process. Furthermore, searches of Google Books and certain other databases which provide small amounts of citation data (e.g., Psyc/NFO) may turn up still more unique citing references.

At least one scholar has expressed concern that metrics such as Web of Science's Impact Factor do not accurately reflect the true influence of social work journals within the discipline partially due to the narrow, two year time period for gathering citations, and partially due to the fact that many critical journals are left out of the ranking and calculations. ${ }^{38}$ Scopus tracks citation data for a greater number of journals than Web of Science. Furthermore, Scopus makes accessible its journal influence measures (SNIP and SJR) for all journals in the database, whereas Web of Science provides the JIF for only the highest scoring journals in the field. As a result, Scopus may prove to be a more informative and robust source of information on scholarly influence in social welfare than Web of Science.

The utility of Google Scholar data is contingent upon several factors. As a supplement to Web of Science and Scopus, it can provide more robust information and coverage. Ball and Tunger asserted that researchers now need to justify their reasons for using either Science Citation Index or Scopus in their analysis. ${ }^{39}$ If using Google Scholar, the researcher must go even further - he or she needs to determine (and justify) if Google Scholar should be utilized, but also whether to include the myriad non-traditional 
document types that appear in Google Scholar, and whether those formats are deemed scholarly, or at least of sufficient quality to demonstrate influence or impact. The justification of what types of resources are used may vary based on the academic discipline, type of bibliometric measurements used, and the overall purpose for the analysis.

This study looked at both the total number of citing references retrieved from Google Scholar as well as only those citing references which were from scholarly journal articles. As was shown, Google Scholar provided vastly larger citation counts than either Scopus or Web of Science when all results are taken into account, but only slightly larger counts when only scholarly journals are considered. The natural question that comes out of this discovery is that of scholarly rigor. What should and should not be considered a scholarly source of citations? Researchers have published studies indicating that online syllabi, PowerPoint presentations, and scholarly blogs should be considered along with scholarly journals as markers of influence or impact. ${ }^{40}$ In addition, at least one scholar has noted that the omission of citations from non-English language sources is a tremendous oversight. ${ }^{41}$ For social welfare, it would appear that a large number of sources in Google Scholar would be considered scholarly - articles, books, and dissertations are the top three document formats of citing references to the journals selected for this study. Therefore, using Google Scholar is a useful addition to the social welfare researcher's arsenal of tools for measuring scholarly communication, journal use, and influence. Nonetheless there is a further caveat that should be acknowledged: Beel and Gipp have determined that with a little web coding knowledge, it is relatively easy to falsify citing references to research and create "search engine spam" which artificially inflates citation counts within Google Scholar ${ }^{42}$ While it is unclear as to whether this is occurring deliberately and if so, to what extent, it remains an issue which should engender cautious use of search engine citation data. Unfortunately, at this time the only way to guard against this type of false hit is to evaluate the citing references from Google Scholar by looking at each one individually. 


\section{Conclusion}

It has been asserted that it is sufficient to track citations only to those journals which are "most important," usually based on their past citation rates. ${ }^{43}$ While there is something to be said for focusing on citations from publications that have been evaluated for "importance," it may simply not be sufficient in the new era of electronic publishing, open access, and web documents. With the advent of new tools which include more than just citing references from journals, Web of Science may be sufficient for creating a ranking of journals based on citation count, but it is the least comprehensive if used to locate the greatest number of citing references. If one values the quality review of content included in a citation database, Scopus may be preferred to Web of Science for social welfare journals for its higher citation counts; however both databases should be used if possible. Google Scholar provides useful results, but due to the lack of quality review, citation counts do not follow a pattern similar to the other two databases. Furthermore the considerations of document type, quality review, and the possibility of "search engine spam" need to be understood and accounted for before incorporating Google Scholar data. As a result, Google Scholar may not be as reliable as either Scopus or Web of Science as a standalone source for citation data. Nonetheless, to obtain the most comprehensive citation count, one must use all three resources.

\section{$\underline{\text { Acknowledgements }}$}

Many thanks to Zhuocao Liang for her invaluable work in capturing the citation data and providing the preliminary analysis in an organized fashion; Mary Casserly, Mary Jane Brustman, and Mary Van Ullen for their critiques and suggestions, and Cary Gouldin for her detailed proofreading.

\section{Role of the Funding Source}


This research was partially funded by a grant from the Research Foundation of the State University of New York through a University at Albany Faculty Research Awards Program-B grant. This grant funded a graduate student hired as a data entry and analysis assistant. This assistant worked with the author to capture the raw data and provide preliminary organization and analysis of the captured data and for no other purposes.

\footnotetext{
${ }^{1}$ Bruce A. Thyer, “Developing Discipline-Specific Knowledge for Social Work: Is It Possible?” (Council on Social Work EducationCouncil on Social Work Education, Winter 2002).

${ }^{2}$ Ibid., 110.

${ }^{3}$ M. Strothmann, "Use of Non-Social Work Journals in Social Work Research: Results of a Citation Analysis," Behavioral and Social Sciences Librarian 29, no. 4 (2010): 244-266; Y. Berman and A.S. Eaglstein, "The Knowledge Base of Social Work: A Citation Analysis," Aslib Proceedings: New Information Perspectives 46, no. 9 (1994): 225230; Kam-fong Monit Cheung, "Interdisciplinary Relationships Between Social Work and Other Disciplines: A Citation Study," Social Work Research \& Abstracts 26, no. 3 (1990): 23.

${ }^{4}$ Sydney J. Pierce, "Disciplinary Work and Interdisciplinary Areas: Sociology and Bibliometrics," in Scholarly Communication and Bibliometrics (Newbury Park, Calif: SAGE Publications, 1990), 46-58.

${ }^{5}$ Gary Holden, Gary Rosenberg, and Kathleen Barker, Bibliometrics in Social Work (Psychology Press, 2006).

${ }^{6}$ Strothmann, "Use of Non-Social Work Journals in Social Work Research."

${ }^{7}$ Robert G. Green and Mary Secret, "Publishing by Social Work Scholars in Social Work and Non-Social Work Journals," Social Work Research 20, no. 1 (March 1996): 31-42.

${ }^{8}$ H. F Moed, Citation Analysis in Research Evaluation, Information Science and Knowledge Management v. 9

(Dordrecht ; [Great Britain]: Springer, 2005), 44-45.

${ }^{9}$ L. Allen Furr, "The Relative Influence of Social Work Journals: Impact Factors Vs. Core Influence," Journal of Social Work Education 31, no. 1 (1995): 38-45.

${ }^{10}$ Anne-Wil Harzing, The Publish or Perish Book: Your Guide to Effective and Responsible Citation Analysis, 1st ed. (Melbourne: Tarma Software Research Pty Ltd, 2010), 220-221.
} 
${ }^{11}$ L.I. Meho and Y. Rogers, “Citation Counting, Citation Ranking, and H-index of Human-Computer Interaction Researchers: A Comparison of Scopus and Web of Science," Journal of the American Society for Information Science and Technology 59, no. 11 (2008): 1711-1726; L.I. Meho and C.R. Sugimoto, “Assessing the Scholarly Impact of Information Studies: A Tale of Two Citation Databases-Scopus and Web of Science," Journal of the American Society for Information Science and Technology 60, no. 12 (2009): 2499-2508; L.I. Meho and K. Yang, “Impact of Data Sources on Citation Counts and Rankings of LIS Faculty: Web of Science Versus Scopus and Google Scholar," Journal of the American Society for Information Science and Technology 58, no. 13 (2007): 2105-2125.

12 Meho and Sugimoto, "Assessing the Scholarly Impact of Information Studies."

${ }^{13}$ Meho and Rogers, "Citation Counting, Citation Ranking, and H-index of Human-Computer Interaction Researchers."

${ }^{14}$ S. Mikki, "Comparing Google Scholar and ISI Web of Science for Earth Sciences," Scientometrics 82, no. 2 (2010): $321-331$.

${ }^{15}$ Ibid., 330.

${ }^{16}$ M. Levine-Clark and E.L. Gil, "A Comparative Citation Analysis of Web of Science, Scopus, and Google Scholar," Journal of Business and Finance Librarianship 14, no. 1 (2009): 32-46.

${ }^{17}$ Abhaya V Kulkarni et al., "Comparisons of Citations in Web of Science, Scopus, and Google Scholar for Articles Published in General Medical Journals," JAMA: The Journal of the American Medical Association 302, no. 10 (September 9, 2009): 1092-1096.

${ }^{18}$ G. Haddow and P. Genoni, "Citation Analysis and Peer Ranking of Australian Social Science Journals," Scientometrics 85, no. 2 (2010): 471-487.

${ }^{19}$ M. Norris and C. Oppenheim, "Comparing Alternatives to the Web of Science for Coverage of the Social Sciences' Literature," Journal of Informetrics 1, no. 2 (2007): 161-169.

${ }^{20}$ L. Bornmann et al., "Convergent Validity of Bibliometric Google Scholar Data in the Field of Chemistry-Citation Counts for Papers That Were Accepted by Angewandte Chemie International Edition or Rejected but Published Elsewhere, Using Google Scholar, Science Citation Index, Scopus, and Chemical Abstracts," Journal of Informetrics 3, no. 1 (2009): 27-35. 
${ }^{21}$ J. Bar-Ilan, "Citations to the 'Introduction to Informetrics' Indexed by WOS, Scopus and Google Scholar," Scientometrics 82, no. 3 (2010): 495-506.

${ }^{22}$ É. Archambault et al., "Comparing Bibliometric Statistics Obtained from the Web of Science and Scopus," Journal of the American Society for Information Science and Technology 60, no. 7 (2009): 1320-1326.

${ }^{23}$ Susanne Mikki, "Google Scholar Compared to Web of Science: A Literature Review," Nordic Journal of Information Literacy in Higher Education 1, no. 1 (March 26, 2009), https://noril.uib.no/index.php/noril/article/view/10.

${ }^{24}$ S.L. Sellers et al., "Evaluation of Social Work Journal Quality: Citation Versus Reputation Approaches," Journal of Social Work Education 40, no. 1 (2004): 143-160.

${ }^{25}$ Ram A. Caputo Cnaan, "Senior Faculty Perceptions of Social Work Journals," Journal of Social Work Education 30, no. 2 (Spring 1994): 185-199.

${ }^{26}$ Sellers et al., “Evaluation of Social Work Journal Quality,” 148.

27 "Web of Science Field Tags (Articles and Conference Proceedings)," ISI Web of Science Help, 2009, http://images.webofknowledge.com/WOK45/help/WOK/hft_wos.html.

${ }^{28}$ Elsevier B.V., "What Does It Cover? | SciVerse”, n.d., http://www.info.sciverse.com/Scopus/Scopus-indetail/facts.

${ }^{29}$ Elsevier B.V., “CSAB | SciVerse”, n.d., http://www.info.sciverse.com/Scopus/csab.

${ }^{30}$ Thomson Reuters, "Thomson Reuters | The Thomson Reuters Journal Selection Process | Science", n.d., http://thomsonreuters.com/products_services/science/free/essays/journal_selection_process/.

${ }^{31}$ Cnaan, "Senior Faculty Perceptions of Social Work Journals."

${ }^{32}$ Kulkarni et al., "Comparisons of Citations in Web of Science, Scopus, and Google Scholar for Articles Published in General Medical Journals"; Levine-Clark and Gil, "A Comparative Citation Analysis of Web of Science, Scopus, and Google Scholar."

${ }^{33}$ Martin Rosvall and Carl T. Bergstrom, "An Information-Theoretic Framework for Resolving Community Structure in Complex Networks," Proceedings of the National Academy of Sciences, no. 104 (2007): 7327-7331.

${ }^{34}$ Harzing, The Publish or Perish Book. 
${ }^{35}$ Lutz Bornmann and Hans-Dieter Daniel, “What Do Citation Counts Measure? A Review of Studies on Citing

Behavior," Journal of Documentation 64, no. 1 (January 18, 2008): 70.

${ }^{36}$ Ibid., 66-67.

${ }^{37}$ Harzing, The Publish or Perish Book, 221.

${ }^{38}$ Furr, "The Relative Influence of Social Work Journals."

${ }^{39}$ R. Ball and D. Tunger, “Science Indicators Revisited - Science Citation Index Versus Scopus: A Bibliometric Comparison of Both Citation Databases," Information Services and Use 26, no. 4 (2006): 293-301.

${ }^{40}$ Mike Thelwall and Kayvan Kousha, "Online Presentations as a Source of Scientific Impact? An Analysis of PowerPoint Files Citing Academic Journals," Journal of the American Society for Information Science and Technology 59, no. 5 (March 1, 2008): 805-815; K. Kousha, M. Thelwall, and S. Rezaie, “Using the Web for Research Evaluation: The Integrated Online Impact Indicator," Journal of Informetrics 4, no. 1 (2010): $124-135$.

${ }^{41}$ Nicola De Bellis, Bibliometrics and Citation Analysis: From the Science Citation Index to Cybermetrics (Lanham, Md: Scarecrow Press, 2009).

42 Joeran Beel and Bela Gipp, “Academic Search Engine Spam and Google Scholar's Resilience Against It,” Journal of Electronic Publishing 13, no. 3 (December 2010), http://hdl.handle.net/2027/spo.3336451.0013.305.

${ }^{43}$ Moed, Citation Analysis in Research Evaluation.

\section{Captions for Figures}

Figure 1. Patterns of Overlap and Unique Citations (Number and Percentage of Total Citations)

Figure 2. Source Types of All Citing References

Figure 3. Ranking of Journals by Cited Reference Count

Figure 4. Comparison of Citation Count Ranking and Sellers, et al. Ranking

Figure 5. Source Types of Citing References from Each Database to Each Journal

Figure 6. Distribution of Unique Citing References for Each Journal 
Figure 7. Relative Proportion of Citation Coverage: The total citation count for each journal was subtracted from the journal with the highest count, the difference is shown and compared for each citation database. 